\title{
Publisher's Note: Pairing symmetry of the one-band Hubbard model in the paramagnetic weak-coupling limit: A numerical RPA study [Phys. Rev. B 92, 104505 (2015)]
}

\author{
A. T. Rømer, A. Kreisel, I. Eremin, M. A. Malakhov, T. A. Maier, P. J. Hirschfeld, and B. M. Andersen \\ (Received 28 July 2016; published 5 August 2016)
}

DOI: 10.1103/PhysRevB.94.059902

This paper was published online on 8 September 2015 with an incorrect support statement in the Acknowledgments. The third sentence in the Acknowledgments should read as "P.J.H. was supported by NSF-DMR-1407502." The Acknowledgments have been corrected as of 28 July 2016. The Acknowledgments are incorrect in the printed version of the journal. 